\title{
Refugios naturales y artificiales de Murciélagos (Mammalia:Chiroptera) en la selva baja en el Noroeste de Perú
}

\author{
Natural and artificial roosts of Bats (Mammalia: Chiroptera) in the low forest in \\ Northwestern Peru
}

\author{
M. Mónica Díaz ${ }^{1 *}$ y Victor Hugo Linares García²
}

${ }^{1}$ PIDBA (Programa de Investigaciones de Biodiversidad Argentina), Facultad de Ciencias Naturales e Instituto Miguel Lillo, Universidad Nacional de Tucumán-PCMA (Programa de Conservación de los Murciélagos de Argentina), CONICET (Consejo Nacional de Investigaciones Científicas y Técnicas); Fundación Miguel Lillo, Miguel Lillo 205, CP 4000, Tucumán, Tel: 03814331339, Argentina,

Universidad Nacional de la Amazonía Peruana. Sargento Lores 385, lquitos, Perú, victorlinga@yahoo.es

*mmonicadiaz@arnetbiz.com.ar

\begin{abstract}
RESUMEN
Se describen los refugios de murciélagos encontrados en un área de selva amazónica de los alrededores de Iquitos (Departamento Loreto, Perú). Los muestreos en ambientes naturales se realizaron mediante caminatas diurnas y encuestas a pobladores, y en las áreas urbanas y suburbanas fueron diurnos y nocturnos, con visitas a edificios y puentes donde previamente se había registrado la presencia de especímenes. En refugios no modificados se registraron ejemplares apoyados sobre árboles o troncos, en huecos de árboles en pie, dentro de troncos caídos, entre hojas de palmeras, y quebradas en el suelo entre raíces de árboles. Las especies encontradas en este tipo de refugios pertenecen a las familias Emballonuridae y Phyllostomidae. En los refugios modificados se registraron especies de la familia Phyllostomidae en tiendas bífidas, tiendas tipo bote invertido y tipo cónica, y en termiteros. Los refugios artificiales fueron edificios, puentes y una embarcación, y las familias registradas fueron Emballonuridae, Noctilionidae, Phyllostomidae, Molossidae y Vespertilionidae. Especímenes de las especies de los géneros Carollia y Glossophaga se encontraron frecuentemente compartiendo refugios. También se ha observado que algunos refugios eran compartidos por ejemplares pertenecientes a diferentes niveles taxonómicos como especies, géneros, e incluso familias.
\end{abstract}

Palabras claves: Ambientes naturales. Ambientes urbanos. Murciélagos. Refugios.

\begin{abstract}
Here we describe the bat roosts found in an area of the Amazon forest around the locality of Iquitos in Loreto Departament, Peru. Natural habitats were surveyed during the day by walking, and with the help of censuses made to local dwellers; in urban and suburban areas the bats were detected by checking in buildings and bridges where the specimens were previously observed. At natural roosts, not modified by bats, the specimens were observed resting on trees or logs, inside treeholes, fallen trunks, among palm leaves, streams on the floor among roots. The species found in these shelters belonged to the families Emballonuridae and Phyllostomidae. In roosts modified by bats we recorded species of the family Phyllostomidae living on bifid tents, inverted boat tents, and conical tents, as well as in termite mounds. The artificial shelters detected were mostly buildings, some bridges and a vessel, where we have found members of the families Emballonuridae, Noctilionidae, Phyllostomidae, Molossidae and Vespertilionidae. At some localities the roosts were shared by specimens of different species, genera and even families; species that often share shelters are members of genera Carollia and Glossophaga.
\end{abstract}

KEYwords: Bats. Natural habitats. Roosts. Urban habitats.

\section{INTRODUCCIÓN}

Losmurciélagospasanmásdelamitaddelavidaensusrefugios (Kunz 1982, Rodríguez-Herrera 2007), entendiéndose como tales a los espacios que les brindan condiciones propicias para cumplir funciones biológicas como hibernación, reproducción, crianza, descanso, o alimentación; a la vez, ofrecen protección contra depredadores y proporcionan un ambiente estable (Kunz 1982). Los refugios pueden ser naturales o artificiales; los primeros incluyen, por ejemplo, 
plantas, cuevas, grietas, y termiteros, mientras que los artificiales están vinculados a construcciones humanas. Algunos murciélagos neotropicales viven en refugios naturales no modificados por ellos mismos (e.g. Artibeus lituratus Olfers) mientras que otros tienen la capacidad de modificar hojas y construir tiendas (Rodríguez-Herrera, 2007), que pueden ser ocupadas por las especies que las construyen (e.g. especies del género Dermanura Gervais) como también por usuarios secundarios (e.g. Uroderma bilobatum Peters) (Lim, 1998).

La selección y el uso de refugios están influidos tanto por factores intrínsecos (preferencias por necesidades metabólicas o por sitios descubiertos o cerrados, economía energética en relación al tamaño, organización social, selección sexual) como extrínsecos (forma del refugio, abundancia y disponibilidad de los sitios, riesgo de depredación, entre otros) (Fenton 1970, Kunz 1982).

La destrucción de sus refugios es una de las amenazas que sufren los murciélagos a lo largo de su distribución, siendo uno de los principales factores de declinación de sus poblaciones (Kunz 1982). De allí la importancia de conocer los refugios que ocupa cada especie y sus características, como también cuales especies están capacitadas para construirlos y cuales actúan como ocupantes secundarios.

A pesar de que un alto número de especies de quirópteros neotropicales que utilizan o construyen tiendas habitan en la selva amazónica de Perú, los estudios sobre estos refugios son prácticamente inexistentes para ese país, y la mayoría de ellos se ha enfocado en las regiones del sur (Foster 1992, Kunz 1994, Tello \& Velazco 2003). En este trabajo se describen los refugios encontrados en un área de selva al noreste de Perú, e incluye observaciones realizadas tanto en ambientes naturales como artificiales.

\section{MATERIALES Y MÉTODOS}

Entre diciembre de 2002 y diciembre de 2005, se realizaron muestreos de murciélagos en diversos puntos a lo largo de una carretera que une las localidades de Iquitos y Nauta, en la selva amazónica de Perú. Estos estudios, conducentes a conocer la diversidad de especies de la zona, se realizaron tanto en ambientes naturales, como en zonas urbanas y suburbanas (ver lista de localidades en Apéndice 1), e incluyeron de manera adicional los muestreos de refugios. Para localizar los refugios en áreas naturales se realizaron caminatas diurnas a lo largo de transectas, abiertas con anterioridad para colocar trampas y redes, y realizando observaciones hasta 20 $\mathrm{m}$ hacia cada lado de las transectas. Estos muestreos fueron complementados con encuestas efectuadas a los pobladores de cada sitio de muestreo, con el objetivo de obtener información que pudiera orientar sobre la ubicación de refugios. En las áreas suburbanas y urbanas se visitaron iglesias, edificios escolares, gubernamentales y viviendas particulares para chequear la presencia de murciélagos.

La colecta de los ejemplares durante los muestreos de refugios se realizó mediante redes de niebla extendidas en los casos donde era posible (Figs. 1A, B), o sosteniendo pequeños tramos de redes en las salidas de los refugios (Fig. 1C); en algunos casos se utilizaron redes entomológicas de tela para facilitar la captura de los individuos (Fig. 1D).

En cada refugio se intentó colectar el total de ejemplares que los habitaban; de cada ejemplar colectado se tomaron datos sobre medida del antebrazo, sexo, condición reproductiva y edad. Los ejemplares colectados fueron depositados en la Colección Mamíferos Lillo (CML) de la Universidad Nacional de Tucumán y Fundación Miguel Lillo, Argentina, y en la Colección de Mamíferos del Museo de Historia Natural de la Universidad Nacional de San Marcos (MUSM), Lima, Perú. Los ejemplares no catalogados aún en las colecciones mencionadas se indican con el número de campo antepuesto por el acrónimo MMD (M. Mónica Díaz), correspondiente al catálogo de la coleccionista.

\section{RESULTADOS}

A continuación se detallan las especies y cantidad de ejemplares examinados en cada tipo de refugio, indicándose en cada uno localidad de ubicación del refugio, cantidad de ejemplares, número de colección, sexo (condición reproductiva) y edad, tipo de refugio donde fue encontrado y fecha de colecta. Se agregan comentarios referidos a aspectos destacables que agregan información sobre cada especie o ejemplar, como ser datos que corresponden a ejemplares que fueron liberados, entre otros.

Dentro de los refugios naturales se pueden mencionar los no modificados (estructuras naturales no modificados por los murciélagos como hojas, troncos, raíces, quebradas, y otros) y los modificados (hace referencia a aquellos refugios construidos a partir de plantas modificadas y a los termiteros). Los refugios artificiales son los que corresponden a las construcciones humanas. En este estudio, a pesar de la gran cantidad de ejemplares colectados en zonas urbanas (612) y rurales (1079), donde la presencia de refugios artificiales es alta, en este trabajo sólo se incluyen a aquellas especies de las cuales se colectaron ejemplares de manera directa del refugio, o de aquellas cuyos refugios fueron identificado fehacientemente. En el caso de iglesias y establecimientos educativos y de salud, las búsquedas fueron realizadas por el equipo de trabajo, pero en la mayoría de las casas particulares y alcantarillas la colecta se hizo como consecuencia de información recibida por parte de vecinos. 


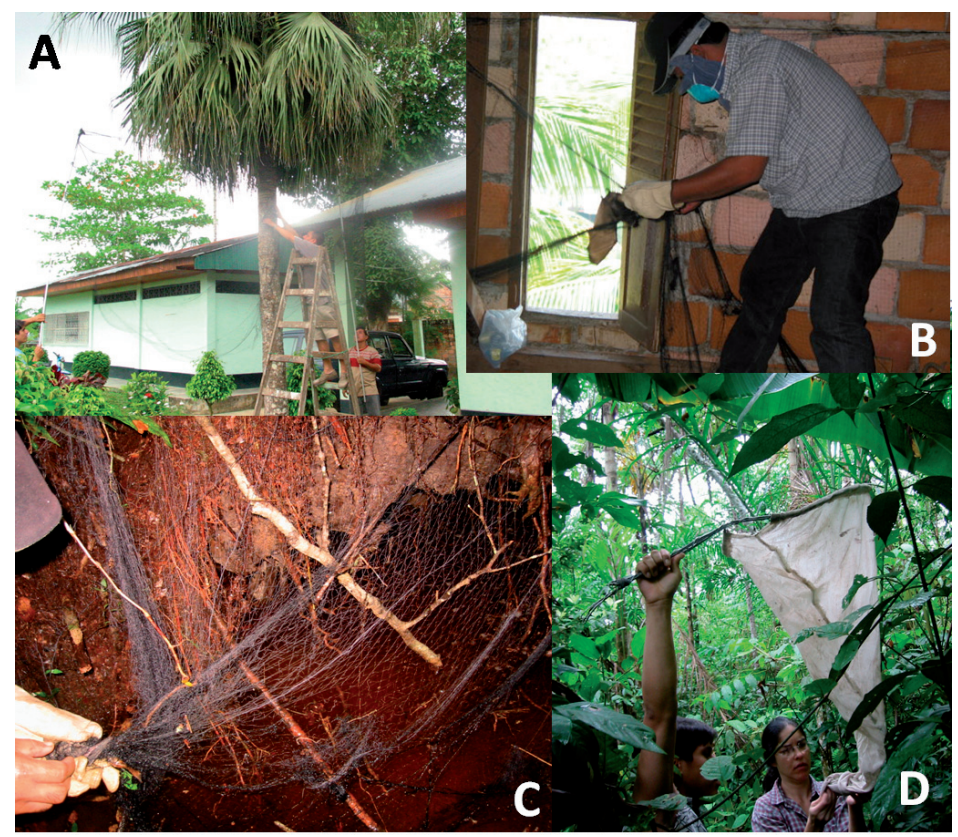

Figura 1. Colecta de ejemplares: A) con redes de niebla fuera del refugio; B) con redes de niebla en el interior del refugio; C) usando pequeños tramos de redes en las salidas de los refugios; D) con redes entomológicas fabricadas en tela.

FIGURE 1. Collection of specimens: A) using mistnet outside the roost; B) using mistnet inside the roost; C) using small pieces of mistnet in the exists of roost; D) using entomological nets.

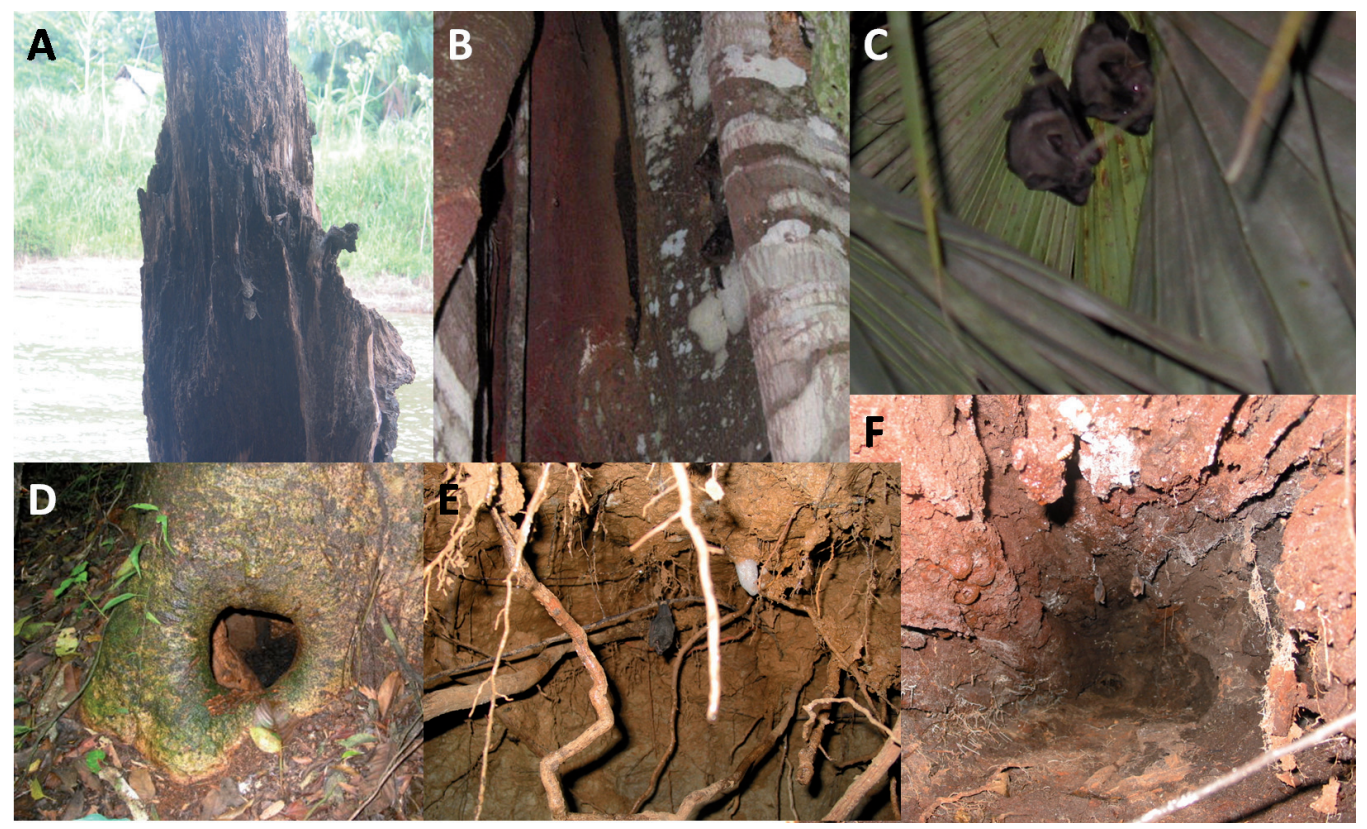

Figura 2. Refugios naturales no modificados. A) Rhynchonycteris naso sobre tronco seco; B) Saccopteryx bilineata sobre tronco de un árbol de Capinurí, Maquira coriacea; C) Artibeus planirostris en hojas de aguaje (Mauritia flexuosa); D) Hueco en la base de un tronco de Catahuaca blanca (Hura crepitans) donde se colectó un ejemplar de Carollia brevicauda; E) Carollia brevicauda en quebrada en el suelo entre raíces de árboles; F) Micronycteris cf. megalotis en el interior de un tronco hueco.

FIGURE 2. Unmodified natural roosts. A) Rhynchonycteris naso on a dry trunk; B) Saccopteryx bilineata on a tree trunk of Capinuri, Maquira coriacea; C) Artibeus planirostris in leaves of aguaje palm (Mauritia flexuosa); D) Tree cavity at the base of white Catahuaca (Hura crepitans) where a specimen of Carollia brevicauda was collected; E) Carollia brevicauda in a large cavity on the ground, transversed by many roots of several trees; F) Micronycteris cf. megalotis inside a hollow log. 


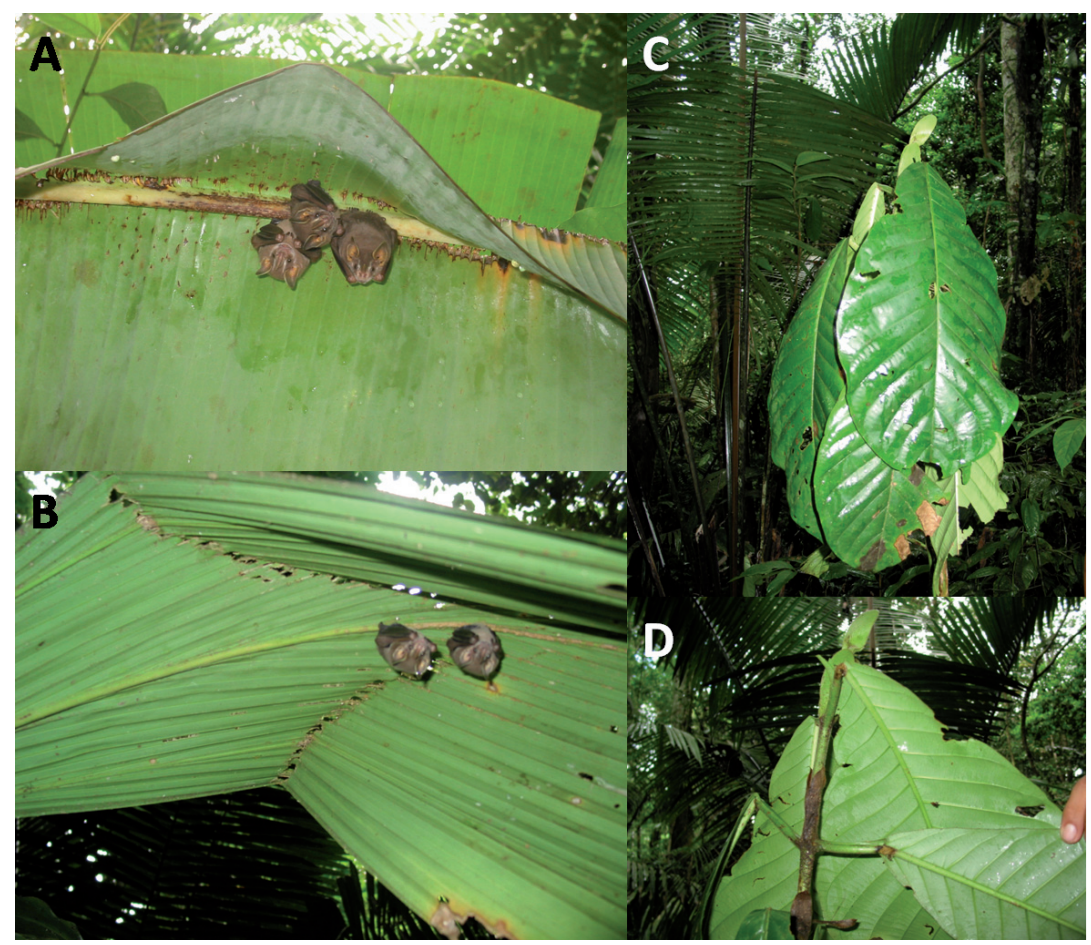

Figura 3. Refugios naturales modificados. A) Dermanura anderseni en tienda tipo bote invertido en hoja de plátano (Musa paradisiaca). B) Dermanura sp. en tienda bífida en Asplundia sp. C) Tienda tipo cónica en Bathysa sp. D) Detalle del corte del pecíolo en la base de las hojas de Bathysa sp.

Figure 3. Natural roosts modified. A) Dermanura anderseni in inverted boat tent in banana leaf (Musa paradisiaca). B) Dermanura sp. in bifid tent in Asplundia sp. C) Conical tent in Bathysa sp. D) Detail of cutting on the petiole at the base of the leaves of Bathysa sp.
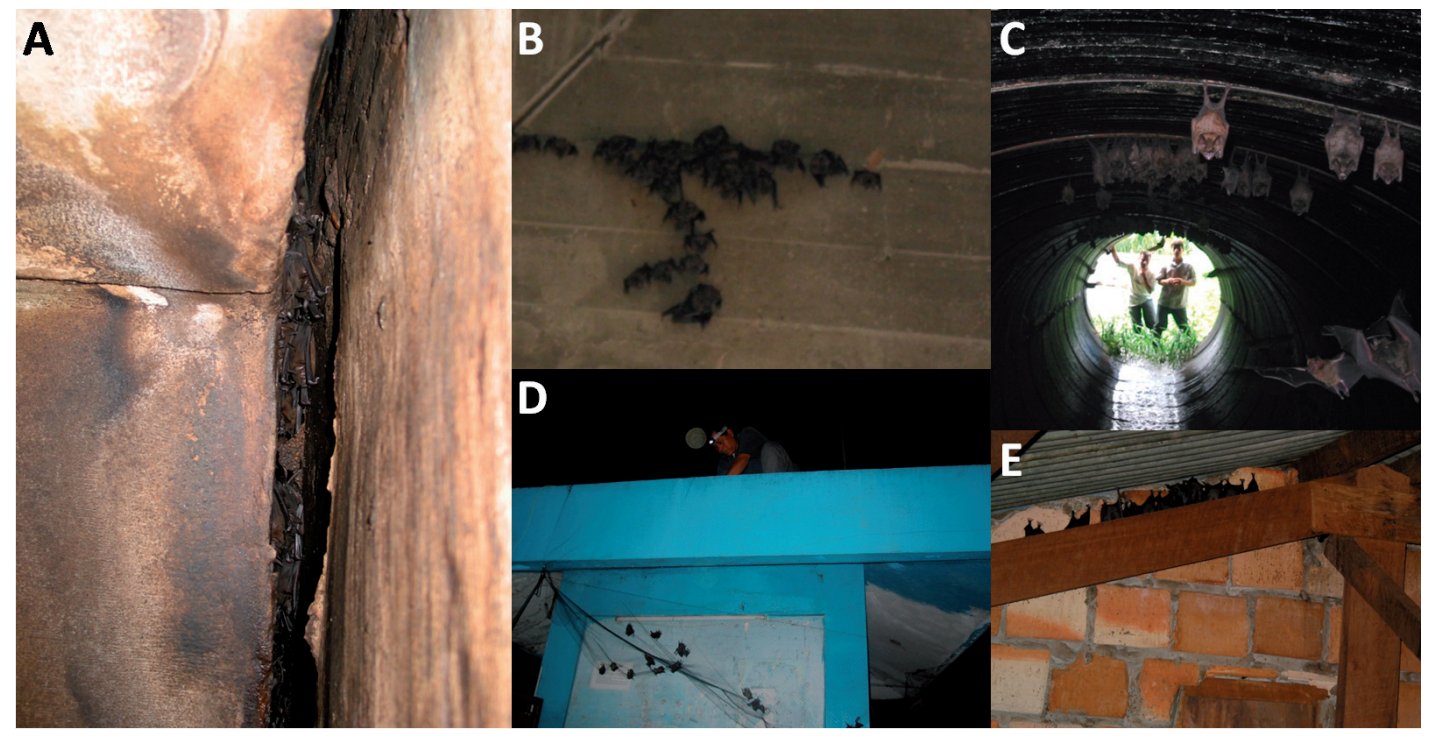

Figura 4. Refugios artificiales. A) Grieta en puente de concreto donde se alojan ejemplares de Noctilio albiventris; B) Colonia de Artibeus planirostris en cúpula de iglesia. C) Alcantarilla con ejemplares de Carollia perspicilliata. D) Colecta de ejemplares de una colonia de Molossus molossus alojados en el techo del camal municipal; E) Phyllostomus hastatus en el altillo de una casa.

Figure 4. Artificial roosts. A) Fissure in concrete bridge where specimens of Noctilio albiventris were housing; B) Colony of Artibeus planirostris at dome of the church. C) Culvert with specimens of Carollia perspicilliata. D) Specimens collected from a colony of Molossus molossus housing in a roof of the Municipal slaughterhouse; E) Phyllostomus hastatus in the attic of a house. 


\section{FAMilia EmBaLlonURIDAE Gervais 1856}

Cormura brevirostris Wagner 1843

Especímenes examinados (2): El Dorado, km 25 de la carretera Iquitos-Nauta, app. 1,5 km al E, 1 (MMD 1816, macho adulto) (en hueco de un árbol, 16 julio 2003); Peña Negra, $600 \mathrm{~m}$ al W del km 10 de la carretera Iquitos-Nauta, 1 (MMD 1409, hembra adulta) (en hueco de un árbol, 20 mayo 2003).

ComentaRIos: Los ejemplares examinados fueron colectados con una red colocada en la salida de un hueco de un árbol vivo; las capturas ocurrieron a las 19 y 20 hs respectivamente; el MMD 1816 en bosque primario y el MMD 1409 en purma (bosque secundario). Esta especie suele refugiarse en huecos de árboles, cavidades superficiales en la base de árboles vivos y puentes (Bernard 2003).

Peropteryx leucoptera Peters 1967

Especímenes eXaminados (2): San Lucas, W km 43 de la carretera Iquitos-Nauta, 2 (MMD 4271, 4272, una hembra preñada y un macho adulto) (en tronco hueco caído, 10 febrero 2005).

Comentarios: Eisenberg (1989) y Hood y Gardner (2008) reportaron que Peropteryx leucoptera se refugia en cuevas, en troncos huecos podridos en el suelo, espacios oscuros bajo grandes árboles, y en cavidades a lo largo de las orillas de los cursos de agua. Durante nuestro estudio de murciélagos en la región, a lo largo de casi cuatro años, únicamente se registró esta especie mediante los dos ejemplares capturados en su refugio. El refugio consistía en un tronco hueco caído, abierto por la mitad y alojaba tres individuos, uno de los cuales no pudo ser capturado, pero sí observado.

Peropteryx pallidoptera Lim et al. 2010

Especímenes examinados (5): El Paujil, 1,8 km al W del km 35 de la carretera Iquitos-Nauta, 5 (MMD 3530-3534; 5 hembras, 2 preñadas) (quebrada en el suelo entre raíces de árboles, 13 julio 2004).

Comentarios: Los ejemplares fueron colectados en una quebrada profunda erosionada por el agua; seis se encontraban refugiados entre raíces expuestas por la erosión del agua, y uno de ellos no pudo ser colectado. En el mismo refugio se capturó un ejemplar de Carollia brevicauda (Schinz).

Rhynchonycteris naso (Wied-Neuwied 1820)

Especímenes examinados (10): Comunidad Puerto Alegría, Río Itaya, 1 (MMD 5111; hembra adulta) (sobre un tronco en la orilla del río Itaya, 8 diciembre 2005) (Fig. 2A); Corrientillo, km 6 de la carretera Iquitos-Nauta, 4,5 km W camino a Zungarococha, 4 (MMD 1592, 1594, 1600,
1602; 1 hembra preñada y 3 machos adultos) (en puente de concreto, 10 y 12 junio 2003); Río Nanay, Puerto Transelva, 5 (MMD 3144-3148; 3 hembras y 2 machos adultos) (en un barco, 2 abril 2004).

Comentarios: Esta especie suele registrarse asociada a cursos de agua, cerca o sobre cursos de aguas lentas, sobre troncos o raíces que sobresalen del agua en zonas muy iluminadas (Plumpton \& Knox Jones 1992), tal como fue registrada en esta oportunidad, sobre un tronco en un área muy iluminada. Se observaron dos ejemplares, pero solo uno de ellos fue colectado. Los ejemplares de Río Nanay fueron observados apoyados sobre las paredes externas de un barco, unos sobre la proa y otros en la popa, en el interior en la sala de máquinas se colectaron ejemplares de Carollia perspicillata. El barco estaba anclado en un tramo del río con selva secundaria. Los ejemplares del puente de concreto fueron colectados con una red de niebla, en la cual también se capturadon Myotis albescens (É. Geoffroy SaintHilaire); los especímenes se podían observar en las uniones de los parantes de hierro de la base del puente y la pared de los pilares.

\section{Saccopteryx bilineata Temminck 1838}

Especímenes examinados (9): Comunidad de Manacamiri, Río Nanay, Fundo Morropón, 5 [MMD 3251-3255, 2 hembras adultas (1 lactando), 3 machos (2 adultos y 1 cría)] [sobre el tronco de un Capinurí, Maquira coriacea (Karsten) (familia Moraceae), 4 mayo 2004]; Ex Petroleros, $300 \mathrm{~m} \mathrm{~W}$ $\mathrm{km} 39,8$ de la carretera Iquitos-Nauta, 4 (MMD 4820-4823, 3 hembras y 1 macho adultos) (sobre la aleta de un tronco de un Capinurí, 12 junio 2005).

Comentarios: Esta especie comúnmente descansa en áreas muy iluminadas en la parte externa de grandes árboles en raíces de apoyo, tipo solapas (Fig. 2B), o en cavidades formadas por estas raíces de soporte (MacCraken \& Wilkinson 2000, Simmons \& Voss 1998, Voigt et al. 2008). A diferencia de otras especies de murciélagos $S$. bilineata se apoya sobre superficies verticales y no en superficies horizontales (Yancey et al. 1998). De los 16 ejemplares obtenidos durante este estudio, nueve fueron encontrados apoyados sobre troncos, en áreas bien iluminadas de bosque primario. En varias localidades se registraron ejemplares sobre troncos durante la búsqueda de refugios, pero la altura en la que se encontraban hacia dificultosa su captura.

\section{FAMilia Noctilionidae Gray 1821}

\section{Noctilio albiventris Desmarest 1818}

EsPeCímenes EXAMINADOS (16): Iquitos Oeste, CESM (Centro Educativo San Martín) "Loreto", 16 [1 CML 7627, 15 MMD 1694, 1705-1713, 1715, 1717-1720; 9 hembras 
adultas (1 preñada, 7 lactando), 7 machos adultos] (puente de concreto, 30 junio 2003).

Comentarios: El refugio se identificó observando la salida de los murciélagos, a las 18:00 hs, los que fueron colectados con redes colocadas en el patio de un colegio cercano al puente. Los especímenes se alojaban en espacios disponibles entre dos pilares de apoyo del puente; las paredes de los pilares estaban cubiertas de heces con fuerte olor (Fig. 4A). Mientras se realizaba el muestreo se observaron muchos ejemplares saliendo durante varios minutos. Esta especie se refugia en árboles huecos y edificios a diferencia de $N$. leporinus que prefiere zonas rocosas y cuevas (Hood \& Pitocchelli 1983).

\section{Familia Phyllostomidae Gray 1825}

\section{Dermanura anderseni (Osgood 1916)}

Especímenes examinados (2): 13 de Febrero, Fundo Nemith, E km 33 de la carretera Iquitos-Nauta, 2 (1 MMD 3746, 1 liberado; hembras preñadas) [tienda tipo bote invertido (Fig. 3A) en plátano (Musa paradisiaca, Musaceae), 11 octubre 2004].

Comentarios: En total se registraron tres ejemplares (Fig. $3 \mathrm{~A}$ ), pero solo se pudieron colectar dos. En la hoja donde se colectaron los especímenes se pudo observar los cortes paralelos a la nervadura central en hojas, típicos en este tipo de tienda (ver Kunz \& Lumsden 2003).

\section{Dermanura sp.}

Especímenes examinados (0): Paujil, W km 37,45 de la carretera Iquitos-Nauta (dos ejemplares fueron observados, pero no colectados) (tienda bífida en Asplundia sp. (Cyclanthaceae), 17 junio 2005).

Comentarios: Lamentablemente solo se obtuvieron registros fotográficos de los ejemplares refugiados en este refugio (Fig. 3B). Las tiendas bífidas se forman por cortes en hojas que terminan en dos puntas; los murciélagos hacen cortes en forma de "J" o "V" a ambos lados de la vena central y las puntas caen formando una cavidad (Kunz et al. 1994); en este caso la hoja tenía un corte en forma de "V", y como es típico se registró en una planta de la familia Cyclanthaceae en el sotobosque de bosque primario (Melo et al. 2009). Los ejemplares observados podrían pertenecer a la especie Dermanura cinereus de acuerdo a los registros citados hasta el momento (ver Rodríguez-Herrera et al. 2007).

\section{Artibeus planirostris (Spix 1823)}

EsPeCímenes examinados (8): Estación Experimental Agraria San Roque, 2 (MMD 3588, 3589, 2 hembras adultas) [entre las hojas de aguaje (Mauritia flexuosa, Arecaceae), 23 agosto 2004]; Iglesia Inmaculada, 6 [5 MMD 3072-3076,
1 liberado; 3 hembras (1 preñada, 1 lactando), 1 macho adulto, 1 feto o cría encontrado muerto, 1 hembra con cría (liberadas)] (en pared y techo de una cúpula de cemento, 18 y 19 marzo 2004).

Comentarios: Artibeus planirostris suele refugiarse en árboles, y se han capturado cerca de árboles frutales (Barquez et al. 1991, 1993, Hollis 2005). Los ejemplares de la Estación Experimental fueron colectados perchando durante el día en una palmera (Fig. 2C), en un parche de vegetación en la ciudad de Iquitos; el grupo estaba conformado por cuatro ejemplares, dos fueron colectados. En la iglesia los ejemplares se encontraban en el campanario, divididos en dos grupos, uno en el extremo de la cúpula y el otro en la pared, unos metros más abajo (Fig. 4B); cada grupo estaba formado por aproximadamente 30 ejemplares; se observó un individuo aislado. En esta oportunidad algunos ejemplares eran más oscuros, probablemente por tratarse de individuos jóvenes. El primer día se encontró un ejemplar recién nacido, muerto en el suelo, donde también se observaron muchas semillas y restos de frutas, lo que sugiere que los ejemplares se alimentaban en el refugio. El segundo día se colocaron redes en la base de la cúpula y se colectaron los ejemplares indicados.

\section{Carollia brevicauda (Schinz 1821)}

Especímenes examinados (6): San Lucas, W km 43 de la carretera Iquitos-Nauta, 1 (MMD 4273; macho adulto) (en árbol hueco grande caído, 10 febrero 2005); Mazanillo, 1 (MMD 5040; hembra preñada) (Fig. 2D) [en hueco en la base de un tronco de Catahuaca blanca (Hura crepitans L., Euphorbiaceae), 19 octubre 2005]; $1 \mathrm{~km} \mathrm{E} \mathrm{km} \mathrm{25,3} \mathrm{de} \mathrm{la}$ carretera Iquitos-Nauta (Fundo San Martín), 1 (MMD 4819; macho adulto) (raíces de un árbol caído, 15 junio 2005); Paujil, W km 37,45 de la carretera Iquitos-Nauta, 2 (MMD 4825, 4826; 1 hembra y 1 macho adultos) (tronco hueco, 17 junio 2005); Camino a El Paujil, 1,8 km al W del km 35 de la carretera Iquitos-Nauta, 1 (MMD 3529; macho adulto) (Fig. 2E) (quebrada en el suelo entre raíces de árboles, 13 julio 2004).

Comentarios: Esta especie ha sido registrada sola o compartiendo refugios con otras especies como Glossophaga soricina (Pallas) y Peropteryx pallidoptera. La co-ocurrencia de Carollia y Glossophaga ya fue registrada en diferentes tipos de refugios tanto naturales como artificiales (Fleming 1988, Graham 1988), y en este estudio, en la localidad de Paujil, C. brevicauda y G. soricina se registraron juntas en un árbol grande hueco y caído con un hueco muy profundo.

\section{Carollia perspicillata Linnaeus 1758}

EsPecímENES EXAMINADOS (33): 13 de Febrero, Fundo Nemith, E km 33 de la carretera Iquitos-Nauta, 2 (MMD 3747, 3748; 1 macho y una hembra adultos) (alcantarilla, 11 octubre 
2004); km 19 de la carretera Iquitos-Nauta, 15 (MMD $1607,1609-1614,1620,1621,1623-1626,1630,1633 ; 10$ hembras y 5 machos adultos) (alcantarilla bajo la ruta, 13 junio 2003); Río Nanay, Puerto Transelva, 10 (MMD 31493158 ; 8 hembras y 2 machos adultos) (barco, en interior en la sala de máquinas, 2 abril 2004); Ninarumi, 7,4 km al W y $500 \mathrm{~m}$ al SE del km 6 de la carretera Iquitos-Nauta, 1 (MMD 2964; hembra adulta) (hueco de tronco caído, 20 febrero 2004); Ninarumi, 7,4 km al $W$ y $1 \mathrm{~km}$ al SE del km 6 de la carretera Iquitos-Nauta, 1 (MMD 4828; macho adulto) (raíces de un árbol caído, 18 junio 2005); Ninarumi, $8,4 \mathrm{~km}$ al W del km 6 de la carretera Iquitos-Nauta, 4 (1 MMD 2864, 3 liberados; una hembra adulta, 3 hembras con crías liberadas) (vivienda, 5 febrero 2004).

COMENTARIOS: Al igual que la especie anterior, C. perspicillata también se registró en huecos de árboles compartiendo con G. soricina, en este caso ejemplares adultos de diferente sexo. En la alcantarilla de 13 de Febrero, donde el tubo estaba con bastante agua estancada y barro, se observaron pocos ejemplares, aproximadamente 20 , mientras que en la alcantarilla debajo de la ruta (Fig. 4C), por la que circulaba agua corriente y permitía el ingreso de una persona de pie, se detectaron aproximadamente un centenar de ejemplares. Los ejemplares de Ninarumi, localizados en una habitación en planta baja de una construcción, estaban dispuestos en fila en una ventana; se capturaron nueve ejemplares, todas hembras adultas, y se liberaron tres que portaban sus crías; aparentemente era una colonia de maternidad. En el mismo refugio se encontraron cinco hembras de G. soricina.

\section{Glossophaga soricina (Pallas 1766)}

Especímenes examinados (9): Iquitos, Coliseo Cerrado de Iquitos, 1 (MMD 1553; hembra adulta) (túneles a los costados de la cancha, 4 junio 2003); Ninarumi, 7,4 km al W y $500 \mathrm{~m}$ al SE del km 6 de la carretera Iquitos-Nauta, 1 (MMD 2965; macho adulto) (hueco en un tronco caído, 20 febrero 2004); Ninarumi, 8,4 km al W del $\mathrm{km} 6$ de la carretera Iquitos-Nauta, 5 (MMD 2859, 2860, 2861, 2862, 2863; hembras adultas) (vivienda, 5 febrero 2004); Paujil, W km 37,45 de la carretera Iquitos-Nauta, 2 (MMD 4824, 4827; 1 hembra y 1 macho adultos) (gran tronco hueco caído, 17 junio 2005).

COMENTARIos: Esta especie ocupa diferentes tipos de refugios como cuevas, troncos, edificaciones humanas, y suele formar colonias de distinto sexo, y colonias de maternidad (ver Willig 1983, Fleming 1988, Alvarez et al. 1991) como es el caso registrado en este trabajo, ya que tres de las hembras registradas en Ninarumi, $8,4 \mathrm{~km}$ al $\mathrm{W}$ estaban preñadas. En las dos localidades donde se registraron en refugios naturales esta especie compartía refugio con otra especie, con Carollia brevicauda en Paujil y con C. perspicillata en Ninarumi. Según Graham (1988) en el este de Perú, el 60\% de los sitios de percha de G. soricina los comparte con $C$. perspicillata, y aparentemente esta asociación se debe al beneficio que logran las especies reduciendo el costo en la termorregulación (Alvarez et al. 1991).

\section{Lonchophylla thomasi Allen 1904}

Especímenes examinados (2): Camino a El Paujil, 1,8 km al $\mathrm{W}$ del km 35 de la carretera Iquitos-Nauta, 2 (MMD 3535, 3536; 1 hembra y 1 macho adultos) (tronco hueco caído, 13 julio 2004).

Comentarios: Tres ejemplares colgados en fila fueron observados dentro de un tronco hueco caído, pero sólo se capturaron dos. Emmons \& Feer (1999) sugieren que esta especie se refugia en pequeños grupos dentro de huecos de árboles, lo que se confirma en este estudio.

\section{Lophostoma silvicolum d'Orbigny 1836}

ESPECÍMENES EXAMINADOS (1): Padrecocha, $30 \mathrm{~m}$ de la carretera Shihua, 1 (MMD 5026; macho adulto) (en termitero, 23 septiembre 2005).

Comentarios: Solo se colectó un macho, pero se observaron otros ejemplares en el refugio, seguramente hembras, ya que se conoce que esta es una especie que suelen formar harenes. Esta especie típicamente ocupa termiteros, y en Panamá Kalko et al. (2006) estudiaron la estructura, modificación y disponibilidad de la pecha y determinaron que los machos excavan y mantienen cavidades en el interior de los nidos activos de termitas. El éxito reproductivo de los machos justifica el esfuerzo y el microclima en el nido (cálido y estable) provee un refugio adecuado para la reproducción de las hembras (Dechmann et al. 2005). Por otro lado, el uso de estos nidos reduce la competencia con otras especies de murciélagos en simpatría, y reduce la carga de parásitos (Kalko et al. 2006).

\section{Micronycteris cf. megalotis}

Especímenes examinados (7): Caserío Palo Seco, app. $500 \mathrm{~m} \mathrm{E} \mathrm{km} \mathrm{28,8} \mathrm{de} \mathrm{la} \mathrm{carretera} \mathrm{Iquitos-Nauta,} 6$ (MMD 4815-4818; 1 hembra y 3 machos adultos; 2 liberados y 4 observados no colectados) (Fig. 2F) (tronco hueco, 14 junio 2005); Ninarumi, 7,4 km al W y $500 \mathrm{~m}$ al SE del $\mathrm{km} 6$ de la carretera Iquitos-Nauta, 1 (MMD 2868 macho adulto) (huecos entre las raíces de un árbol desprendido, 6 febrero 2004).

Comentarios: Esta especie puede refugiarse durante el día en huecos en troncos o árboles y en viviendas (Kalko et al. 1996). En Iquitos fue registrada entre raíces y en troncos huecos; en la primera localidad el refugio entre raíces se inundaba después de fuertes lluvias, y en ese sitio se pudieron observar varios ejemplares, pero solo se pudo capturar uno. 
Mimon crenulatum (É. Geoffroy-Saint Hilaire 1803)

ESPECÍMENES EXAMINAdos (1): 7,4 km al W y $1 \mathrm{~km}$ al SE del $\mathrm{km} 6$ de la carretera Iquitos-Nauta, 1 (MMD 4829; macho adulto) (en tronco de pihuayo (Bactris gasipaes Kunth, Arecaceae), 18 junio 2005).

Comentarios: Sólo un ejemplar de esta especie fue colectado durante este estudio, en un refugio en el interior de un pihuayo, ubicado en un pihuayal, donde se observaron dos ejemplares. Algunos autores (Handley 1976, Kalko et al. 1996, Reid 1997) reportaron a esta especie en huecos de árboles, troncos podridos y tocones de árboles huecos, árboles moribundos abiertos arriba, y también en edificios.

Phyllostomus elongatus (É. Geoffroy Saint Hilaire 1810) ESPECímENES EXAMINADOS (1): Iberia, río Tapiche, 1 (MMD 4916, macho adulto) (en hueco de un árbol "Capinurí": Maquira coriacea, familia Moraceae, 21 Julio 2005).

Comentarios: Varios ejemplares fueron observados en el interior del árbol, pero sólo se pudo colectar uno de ellos; el hueco era muy grande y se podía acceder desde abajo a medio metro del suelo. Aunque este es un refugio conocido para la especie, y se sabe que suele refugiarse en árboles huecos y en huecos de árboles en pie, también se ha registrado en cuevas y edificios.

\section{Phyllostomus hastatus (Pallas 1767)}

Especímenes examinados (23): Caserío Palo Seco, 2,9 km E del $\mathrm{km} \mathrm{28,8}$ de la carretera Iquitos-Nauta, 2 (MMD 4813, 4814; machos adultos) (en tronco de "pijuayo" Bactris gasipaes, Arecaceae, 13 Junio 2005); Rumococha, 3,3 $\mathrm{km} \mathrm{W}$ de Av. Quiñones 4700, 14 (12 MMD 2426-2437; 9 hembras preñadas, 3 machos adultos; 2 liberados) (altillo de una casa, techo a dos aguas, 17-18 de octubre de 2003); Varillal, $400 \mathrm{~m} \mathrm{~W} 200 \mathrm{~m} \mathrm{~N}$ del km 14 de la carretera IquitosNauta, 7 (MMD 1143, 1171, 1179, 1180, 1184, 1193, 1194; machos adultos) (techo de paja de una maloca en un chacra; 24, 25, 27 de marzo de 2003).

Comentarios: Santos et al. (2003) indicaron que esta especie se puede refugiar en huecos de árboles, como es el caso de los ejemplares mencionados del Caserío Palo Seco, colectados en un tronco hueco de pijuayo cerca del camino y de las casas de la villa; un vecino del lugar nos informó sobre la presencia de los murciélagos en el tronco. Santos et al. (2003) también mencionaron que es frecuente encontrar ejemplares de esta especie en construcciones humanas, donde forman harenes de varias hembras con un macho, y grupos de machos separados. Los ejemplares de Rumococha se encontraron alojados en la unión de la pared con el techo de chapa (Fig. 4E) de una habitación, y en el piso se encontraron restos de frutos de tamaño considerable. La mayoría de los especímenes presentaban áreas del cuerpo sin pelos, probablemente causadas por alguna enfermedad o parasitismo similar a la sarna de animales domésticos; también llamaba la atención la presencia de manchas de albinismo en la mayoría de los ejemplares. Junto a estos especímenes se colectó un ejemplar de Molossus molossus (Pallas), asociación registrada también en Brasil por Costa et al. (2010). Los especímenes de Varillal se refugiaban entre las palmas del techo de paja de una "maloca" (construcción comunitaria con techo de palma).

\section{Familia Molossidae Gervais 1856}

Molossus coibensis Allen 1904

Especímenes examinados (10): Iquitos Colegio Primario Secundario "RADM" (Rosa Agustina Donayre de Morey), calle Putumayo, 4 (1 CML 75623, 3 MMD 4336, 4337, 4341; 3 hembras, 1 macho); Iquitos, Edificio EsSalud (calles Raymondi-Napo), 4 (MMD 4864, 4873, 4876, 4878; hembras); Punchana, Calle Amazonas 1086, 2 (1 CML 7563, 1 MMD 3124; 1 macho y 1 hembra adultos) (en el cielorraso de una vivienda, 24 marzo 2004).

Comentarios: Esta especie fue considerada sinónimo de M. molossus por mucho tiempo, por lo que poco se conoce acerca de sus refugios e historia natural. Estos ejemplares fueron registrados junto a gran cantidad de especímenes de M. molossus.

Molossus molossus (Pallas 1766)

Especímenes eXAminados (98): Punchana, Calle Amazonas 1086, 13 (MMD 3114-3123, 3126, 3127, 3128; 10 hembras 7 preñadas y lactando, 2 machos adultos y 1 macho juvenil) (cielorraso de una vivienda, 24 marzo 2004); Rumococha, 3,3 km W de Av. Quiñones 4700, 1 (MMD 2425; macho adulto) (techo de una vivienda, 17-18 octubre 2003); Camal Municipal de Punchana, 25 [22 MMD 3091-3112, 3 liberados; 20 hembras ( 8 preñadas), 4 machos adultos, 1 sexo indeterminado, encontrado como esqueleto] (cielorraso del edificio (Fig. 4D), 23 marzo 2004); CEI Centro Educativo Inicial Nro 165, Republica Federal de Alemania, Club de Leones, 5 (MMD 3067-3071; 4 hembras, 1 macho) (cielorraso del edificio, 17 marzo 2004); Hogar "Padre Angel Rodríguez" (INABIF), Av. 28 de Julio Nro 500, 7 (MMD 3077-3083; 6 hembras y 1 macho) (cielorraso del edificio, 22 marzo 2004); Iquitos (Bermudez 1004), 5 (MMD 1536-1540; 4 hembras y 1 macho juveniles) (entretecho de una vivienda, 2 junio 2003); Iquitos, Calle Roman nro. 988; 10 noviembre 2005, 6 (MMD, 5081-5086; 3 hembras adultas, 1 hembra juvenil, 1 macho adulto, 1 macho juvenil) (entretecho de una vivienda, 10 noviembre 2005); Ninarumi, Colegio Nro 60778, 7,9 km al W del km 6 de la carretera Iquitos-Nauta, 7 (MMD 2852-2858; 3 hembras adultas una lactando, 4 machos adultos) (techo del edificio, 5 febrero 2004); Estación Experimental Agraria San Roque, 21 [MMD 
3564-3584; 10 hembras adultas (8 preñadas), 11 machos adultos] (cielorraso del edificio, 18 agosto 2004); San Juan, Piscina Olímpica del Instituto Peruano de Deportes, km 1,5 al $\mathrm{W}$ de la carretera Iquitos-Nauta, 8 [MMD 2404-2411; 7 hembras adultas, (4 preñadas), 1 macho adulto] (techo del edificio, 9 octubre 2003).

COMENTARIOS: Esta es una especie que comúnmente se registra en zonas urbanas donde se refugia en viviendas humanas. Todos los ejemplares de este estudio, con excepción de uno colectado en bosque secundario, fueron capturados en zonas rurales (16\%) y urbanas (83\%), de los cuales el $60 \%$ de los de zonas rurales y el $69 \%$ de los de las zonas urbanas fueron colectados en sus refugios.

Molossus rufus É. Geoffroy Saint-Hilaire 1805

Especímenes eXaminados (4): Iquitos, Villa de técnicos de la FAP (Fuerza Aérea de Perú), calle Moore última cuadra, 4 (MMD 4957-4960; 4 machos adultos) (cielorraso del edificio, 24 agosto 2005).

Comentarios: En este estudio, todos los ejemplares de esta especie fueron colectados en ambientes urbanos. Los especímenes aquí citados fueron colectados manualmente en el cielorraso de uno de los edificios de la villa, y se pudieron identificar dos grupos de unos 10 ejemplares cada uno. Los edificios eran de tres pisos, techados con hojas de una palmera (irapay, Lepidocaryum tenue Mart., Arecarae), y el área estaba rodeada de vegetación mayormente exótica, ornamental. La mayoría de los ejemplares tenian manchas de albinismo.

\section{Familia Vespertilionidae Gray 1821}

Myotis albescens (É. Geoffroy Saint-Hilaire 1806)

Especímenes examinados (3): Corrientillo, $\mathrm{km} 6$ de la carretera Iquitos-Nauta, 4,5 $\mathrm{km} \mathrm{W}$ camino a Zungarococha, 3 (1 CML 7635, 2 MMD 1591, 1593; 1 hembra preñada y 2 machos adultos) (en puente de concreto, 12 junio 2003).

Comentarios: Los ejemplares pudieron ser observaros por debajo del puente, en unas grietas entre la base y el pilar del puente, los mismos fueron colectados con redes colocadas a ambos lados del puente, sobre el agua; junto a especímenes de esta especie se colectaron ejemplares de Rhynchonycteris naso, que ocupaban el mismo sitio de refugio. Esta especie frecuentemente se refugian en edificios y en otras estructuras humanas, y suelen encontrarse cerca de áreas con agua (Braun et al. 2009).

\section{Myotis riparius Handley 1960}

Especímenes examinados (7): Punchana, Bellavista Nanay (puerto), 4 (MMD 2398, 2399, 2400, 2402; hembras adultas, una preñada y dos lactando) (techo de una vivienda,
8 octubre 2003); Recreo Don Pepe, al lado del cementerio de Punchana, 3 (MMD 3088, 3089, 3090; todas hembras adultas, dos preñadas) (techo de una vivienda, 22 marzo 2004).

Comentarios: Los ejemplares se encontraron en techos de palmas, refugiados entre las hojas, fueron colectando usando redes de niebla a la salida del refugio.

\section{DISCUSION Y CONCLUSIONES}

De las 23 especies registradas mediante la búsqueda de refugios, 15 se capturaron en refugios naturales no modificados (cinco emballonúridos y nueve filostómidos), tres en refugios naturales modificados (filostómidos) y 11 en refugios artificiales (un emballonúrido, un noctiliónido, cuatro filostómidos, tres molósidos y dos vespertiliónidos) (Tabla 1). Solo cinco especies fueron encontradas en los dos tipos de refugios, naturales y artificiales (R. naso, $A$. planirostris, C. brevicauda, G. soricina y P. hastatus). Los censos a pobladores en zonas rurales y naturales, permitió detectar que la mayoría de los habitantes del área conoce lugares donde se refugian los murciélagos, e incluso en dos oportunidades se observaron pobladores que utilizan redes de pesca colocadas en los árboles frutales para colectar murciélagos, debido a la creencia popular que todos los murciélagos son vampiros. En las zonas urbanas, los edificios públicos fueron revisados para buscar ejemplares, pero las colectas en viviendas particulares se realizaron como consecuencia de los llamados de alerta de los propietarios, advirtiéndonos sobre la presencia de murciélagos en sus techos. La ciudad de Iquitos, por estar rodeada de vegetación, soporta permanentemente el desplazamiento de murciélagos entre áreas naturales y urbanas, razón por la cual no solo se colectaron especies insectívoras típicas de ciudades, sino también frugívoras y nectarívoras, típicas de las zonas boscosas.

En algunos de los refugios relevados se descubrieron ejemplares pertenecientes a diferentes especies, géneros y aún familias compartiendo el sitio, pero lo más común fue registrar alguna especie de Carollia (C. brevicauda o $C$. perspicillata) o G. soricina junto a otras especies (Tabla 1). El hecho de compartir refugios provee de beneficios a las especies, por ejemplo las colonias de maternidad reducen su costo de termorregulación, importante en la hembras reproductivas debido a que las bajas temperaturas y el torpor retrasan el desarrollo prenatal y de las crías; por otro lado, la información que se transfiere en los refugios reduce los costos relacionados con la búsqueda de áreas de forrajeo o de otros refugios adecuados, como así también los costos de crianza, riesgos de predación, y selección sexual (Kunz 1982, Willis \& Brigham 2004, Dechmann \& Kerth 2008). 
Kunz \& Lumsden (2003) indicaron que las familias Phyllostomidae y Emballonuridae ocupan principalmente huecos de árboles, lo que fue confirmado para las especies de esas familias en nuestra área de estudio, habiendo encontrado los refugios en huecos de árboles en pie como en troncos huecos caídos. También fue frecuente registrar especies de estas familias entre raíces de árboles caídos o quebradas en el suelo, y también $S$. bilineata y R. naso (Emballonuridae) fueron registradas sobre árboles o troncos, en áreas muy expuestas a la luz, y muchas veces cerca del agua.

TABLA 1. Lista de especies por familias registradas en tres tipos de refugios y especies encontradas compartiendo el mismo sitio.

TABLE 1. List of species by families recorded in three types of roosts and species found sharing the roost.

\begin{tabular}{|c|c|c|c|c|}
\hline & \multicolumn{2}{|c|}{ Refugios Naturales } & \multirow[t]{2}{*}{ Refugios Artificiales } & \multirow[b]{2}{*}{ COMPARTIDO CON } \\
\hline ESPECIES & No MODIFICAdos & Modificados & & \\
\hline \multicolumn{5}{|l|}{ EMBALLONURIDAE } \\
\hline Cormura brevirostris & tronco de árbol en pie & & & \\
\hline Peropteryx leucoptera & tronco hueco caído & & & \\
\hline Peropteryx pallidoptera & $\begin{array}{c}\text { quebrada entre raíces de } \\
\text { árboles }\end{array}$ & & & C. brevicauda \\
\hline Saccopteryx bilineata & sobre un tronco & & & \\
\hline Rhynchonycteris naso & sobre un tronco & & barco, puente & $\begin{array}{c}\text { M. albescens, } C . \\
\text { perspicillata }\end{array}$ \\
\hline \multicolumn{5}{|l|}{ Phyllostomidae } \\
\hline Artibeus planirostris & entre hojas & & cúpula & \\
\hline Carollia brevicauda & $\begin{array}{l}\text { tronco de árbol en pie, } \\
\text { tronco caído, raíces }\end{array}$ & & $\begin{array}{l}\text { alcantarilla, barco, } \\
\text { vivienda }\end{array}$ & $\begin{array}{l}\text { G. soricina, } P \text {. } \\
\text { pallidoptera }\end{array}$ \\
\hline Carollia perspicillata & tronco caído, raíces & & & G. soricina, $R$. naso \\
\hline Dermanura anderseni & & tienda tipo bote & & \\
\hline Dermanura sp & & tienda bífida & & \\
\hline Glossophaga soricina & tronco hueco caído & & vivienda, edificio & \\
\hline Lonchophylla thomasi & tronco hueco caído & & & \\
\hline Lophostoma silvicolum & & termitero & & \\
\hline Micronycteris cf. megalotis & tronco caído, raíces & & & \\
\hline Mimon crenulatum & tronco de árbol en pie & & & \\
\hline Phyllostomus elongatus & tronco de árbol en pie & & & \\
\hline Phyllostomus hastatus & tronco de árbol en pie & & vivienda & M. molossus \\
\hline \multicolumn{5}{|l|}{ Noctilionidae } \\
\hline Noctilio albiventris & & & puente & \\
\hline \multicolumn{5}{|l|}{ Molossidae } \\
\hline Molossus coibensis & & & vivienda & M. molossus \\
\hline Molossus molossus & & & vivienda & P. hastatus, M. coibensis \\
\hline Molossus rufus & & & vivienda & \\
\hline \multicolumn{5}{|l|}{ VESPERTILIONIDAE } \\
\hline Myotis albescens & & & puente & R. naso \\
\hline Myotis riparius & & & vivienda & \\
\hline
\end{tabular}


Las especies registradas en hojas modificadas pertenecen a la familia Phyllostomidae, únicos constructores de tiendas en el Neotrópico (Rodríguez-Herrera et al. 2007). En el área de estudio se registraron numerosas hojas modificadas, pero lamentablemente solo se pudo colectar ejemplares en una oportunidad, y determinar la especie. En la localidad de Paujil, W km 37,45 de la carretera Iquitos-Nauta, se registró una tienda cónica (Bathysa sp., Rubiaceae) (Fig. $3 \mathrm{C}$ ), pero los ejemplares que la utilizaban no pudieron ser identificados. Esta tienda se caracteriza porque los murciélagos realizan cortes en la base de los pecíolos (Fig. 3D) haciendo que las hojas caigan formando un cono (Kunz et al. 1994); este tipo de tienda es construido con plantas de las familias Anacardiaceae, Loganiaceae, Mirsynaceae, Rubiaceae, entre otras (Kunz et al. 1994, Kunz \& Lumsden 2003, Rodríguez-Herrera et al. 2007). Hasta el momento las únicas tres especies de quirópteros registradas en ellas son Uroderma bilobatum, Platyrrhinus helleri (Peters) y Vampyriscus nymphaea (Thomas)(Rodríguez-Herrera \& Tschapka 2005, Rodríguez-Herrera et al. 2007) las dos primeras presentes en la zona de estudio. En otro sitio, San Lucas, W km 43 de la carretera Iquitos-Nauta, se encontraron refugios abandonados en hojas de plátano (Musa paradisíaca L., Musaceae), de irapay (Lepidocaryum tenue Mart., Arecarae) y bombonaje (Cardulovica palmata Ruiz \& Pav., Cyclantaceae) y observó un ejemplar en las hojas de un aguaje (Mauritia flexuosa L.f., Arecaceae). En este sentido se considera que es necesaria la realización de estudios específicos que permitan ampliar el conocimiento sobre los murciélagos tenderos en Perú.

En las zonas rurales y urbanas prácticamente todos los ejemplares colectados en techos y cielorrasos pertenecía a la especie M. molossus. Además se registró una colonia de Noctilio albiventris con numerosos individuos refugiados en un puente de cemento, y en otro puente de menor tamaño se encontraron ejemplares de Myotis albescens y Rhynchonycteris naso; en ambos puentes los ejemplares estaban refugiados en grietas muy pequeñas que dificultaba verlos. Esta misma observación fue destacada por Geluso $\&$ Mink (2009) quienes, en un estudio realizado en México, indicaron que los ejemplares que se refugian en puentes lo hacen en fisuras angostas o grietas; además, mencionaron que la mayoría de sus observaciones de refugios en puentes correspondían a aquellos construidos con vigas, mientras que en puentes construidos con cemento, metal, o con una combinación de ambos pero con grietas escasas, sólo registraron el $0.2 \%$ de sus observaciones.

Finalmente, las especies que usan refugios naturales son más susceptibles de sufrir impactos poblacionales negativos, debido a que el avance de la deforestación y la fragmentación de los ambientes afectan de manera directa sobre sus refugios, y deberían ser las primeras en tenerse en cuenta al momento de pensar en planes de manejo y conservación. Por el contrario, aquellas especies con posibilidades de ocupar ambientes antropizados y refugiarse en viviendas humanas, puentes, u otro tipo de construcciones, tienen posibilidades de hábitat alternativos.

\section{AGRADECIMIENTOS}

Agradecemos a Elvis Valderrama y William Lamar por ayudarnos en la identificación de las plantas donde los refugios fueron encontrados. A Bernal RodríguezHerrera y dos revisores anónimos por sus comentarios y sugerencias que ayudaron a mejorar el manuscrito original. Nuestro más sincero agradecimiento a Sixto Mananita, Harold Portocarrero, Cesar Ahuanari, Rubi Angulo, y a los estudiantes de la Universidad Mayor de San Marcos por su invalorable ayuda durante el trabajo de campo y laboratorio. El trabajo de campo se realizó con subsidios de United States Public Health Service National Institute of Allergy and Infectious Diseases, Estados unidos (R01TW005860, D43TW007120, y K24AI068903). Un especial agradecimiento al Dr. Joseph Vinetz, investigador principal del proyecto desarrollado en Iquitos. También agradecemos al Instituto Nacional de Recursos Naturales (INRENA) y al Ministerio de Agricultura de Perú por los permisos de captura y colecta de ejemplares.

\section{BIBLIOGRAFIA}

Alvarez, J., Willig, M.R., Knox Jones JR., J. \& Webster, W.D. 1991. Glossophaga soricina. Mammalian Species 379:17.

Barquez, R.M., Giannini, N.P. \& Mares, M.A. 1993. Guía de Murciélagos de Argentina. Oklahoma Museum of Natural History, University of Oklahoma. $119 \mathrm{pp}$.

Barquez, R.M., Mares, M.A. \& OJeda, R.A. 1991. Mamíferos de Tucumán - Mammals of Tucumán. Oklahoma Museum of Natural History, University of Oklahoma, Norman. 282 pp.

Bernard, E. 2003. Cormura brevirostris. Mammalian Species 737:1-3.

Braun, J.K., Layman, Q.D. \& Mares M.A. 2009. Myotis albescens (Chiroptera: Vespertilionidae). Mammalian Species 46:19.

Costa, L.M., Lourenço, E.C., Esbérard, C.E.L. \& Silva, R.M. 2010. Colony size, sex ratio and cohabitation in roosts of Phyllostomus hastatus (Pallas) (Chiroptera: Phyllostomidae). Brazilian Journal of Biology 70:10471053.

Dechmann, D.K.N. \& Kerth, G. 2008. My home is your castle: roost making is sexually selected in the bat Lophostoma silvicolum. Journal of Mammalogy 89:1379-1390.

Dechmann, D.K.N., Kalko, E.K.V., König, B. \& Kerth, G. 2005. Mating system of a Neotropical roost-making bat: the 
white-throated, round-eared bat, Lophostoma silvicolum (Chiroptera: Phyllostomidae). Behavioral Ecology and Sociobiology 58: 316-325.

EISENBERG, J.F. 1989. Mammals of the Neotropics. Volume 1. The Northern Neotropics. The University of Chicago Press. 449 pp.

Emmons, L.H. \& Feer, F. 1999. Mamíferos de los bosques húmedos de América tropical: Una guía de campo Santa Cruz, Bolivia: Editorial F.A.N. (Fundación Amigos de la Naturaleza), Bolivia. 298 pp.

Fenton, M.B. 1970. Population studies of Myotis lucifugus (Chiroptera: Vespertilionidae) in Ontario. Life Science Contributions, Royal Ontario Museum 77:1-34.

FLEmING, T.H. 1988. The short-tailed fruit bat: a study in plantanimal interactions. The University of Chicago Press. 365 pp.

Foster, M.S. 1992. Tent roosts of Macconnell's bat (Vampyressa macconnelli). Biotropica 24(3):447-454.

Geluso, K. \& Mink, J.N. 2009. Use of bridges by bats (Mammalia: Chiroptera) in the Rio Grande Valley, New Mexico. The Southwestern Naturalist 54(4):421-429.

Graham, G.L. 1988. Interspecific associations among Peruvian bats at diurnal roosts and roost sites. Journal of Mammalogy 69:711-720.

HandLey, JR. C.O. 1976. Mammals of the Smithsonian Venezuelan Project. Brigham Young University Science Bulletin, Biological Series 20(5):1-91.

Hollis, L. 2005. Artibeus planirostris. Mammalian Species 775:16.

Hood, C.S. \& GARDNer, A.L. 2008. Family Emballonuridae Gervais, 1856. En: Mammals of South America, Volumen I Marsupials, Xenarthrans, Shrews, and Bats (Ed. GARDNER, A.L.), pp. 188-207, The University of Chicago Press.

Hood, C.S. \& Pitocchelli, J. 1983. Noctilio albiventris. Mammalian Species 197:1-5.

Kalko, E.K.V., Handley, C.O. \& Handley, D. 1996. Organization, diversity and long-term dynamics of a Neotropical bat Community. En: Long-term studies in vertebrate communities (Eds. M. Cody \& J. Smallwood). Pp. 503553, Academic Press, Los Angeles.

Kalko, E.K.V., Ueberschaer, K. \& Dechmann, D.K.N. 2006. Roost structure, modification, and availability in the White-throated Round-eared Bat, Lophostoma silvicolum (Phyllostomidae) living in active termite nests. Biotropica 38(3): 1-7.

KunZ, T.H. 1982. Roosting Ecology. En: Ecology of bats (Ed. Kunz, T.H.), pp. 1-56, New York/London: Plenum Press.

Kunz, T.H. 1994. The world of tent-making bats. BATS 12:6-12

Kunz, T.H. \& Lumsden, L.F. 2003. Ecology of cavity and foliage roosting bats. En: Bat Ecology. (Eds.T.H. KUnZ \& M.B. Fenton), pp. 3-90, Chicago: University of Chicago Press.
Kunz, T.H., Fujita, M.S., Brooke, A.P. \& Mccracken, G.F. 1994. Convergence in tent architecture and tent-making behavior among neotropical and pelotropical bats. Journal of Mammalian Evolution 2:57-78.

LiM, B.K. 1998. Relative abundance of small tent-roosting bats (Artibeus phaeotis and Uroderma bilobatum) and foliage tents (Carludovica palmata) in Panama. Bat Research News 39:1-3.

Mccracken, G.F. \& Wilkinson, G.S. 2000. Bat mating systems. En: Reproductive biology of bats (Eds. E.G. CRICHTON \& P. H. Krutzsch), pp. 321-362, Academic, London.

Melo, F.P.L., Rodriguez-Herrera, B., Chazdon, R.L., Medellin, R.A. \& Ceballos, G.G. 2009. Small Tent-Roosting Bats Promote Dispersal of Large-Seeded Plants in a Neotropical Forest. Biotropica 41(6): 737-743.

Plumpton D. L. \& Knox Jones JR. J. 1992. Rhynchonycteris naso. Mammalian Species 413:1-5.

ReID, F. 1997. A field Guide to the Mammals of Central America and Southeast México. Oxford University Press. 334 pp.

Rodríguez-Herrera, B. \& TschapkA M. 2005. Tent use by Vampyressa nymphaea (Chiroptera: Phyllostomidae) in Cecropia insignis (Moraceae) in Costa Rica. Acta Chiropterologica 7:171-174.

Rodríguez-Herrera, B., Medellín, R.A. \& Timm, R.M. 2007. Neotropical Tent-roosting Bats / Murciélagos neotropicales que acampan en hojas. Editorial INBio. Santo Domingo de Heredia, Costa Rica. 184 pp.

Santos, M., Aguirre, L.F., VÁzquez, L.B. \& Ortega, J. 2003. Phyllostomus hastatus. Mammalian Species 722: 1-6.

Simmons, N.B. \& Voss, R.S. 1998. The mammals of Paracou, French Guiana: a neotropical lowland rainforest fauna. Part 1 Bats. Bulletin of the American Museum of Natural History 237:1-219

Tello, J.G. \& Velazco, P.M. 2003. First description of a tent used by Platyrrhinus helleri (Chiroptera: Phyllostomidae). Acta Chiropterologica 5(2):269-276.

Voigt, C.C., Behr, O., Caspers, B., Von Helversen, O., KnÖrnschild, M., Mayer , F. \& Nagy, M. 2008. Songs, scents, and senses: sexual selection in the greater sacwinged bat, Saccopteryx bilineata. Journal of Mammalogy 89:1401-1410.

Willig, M.R. 1983. Composition, microgeographic variation, and sexual dimorphism in Caatingas and Cerrado bat communities from northeast Brazil. Bulletin of Carnegie Museum of Natural History 23:1-131.

Willis, C.K.R. \& Brigham, R.M. 2004. Roost switching, roost sharing and social cohesion: Forest-dwelling big brown bats (Eptesicus fuscus) conform to the fission-fusion model. Animal Behaviour 68: 495-505.

Yancey II, F.D., Goetze, J.R. \& Jones, C. 1998. Saccopteryx bilineata. Mammalian Species 581:1-5. 
Refugios de Murciélagos en la selva del noroeste de Perú: M. Mónica Díaz \& Víctor Hugo Linares

\section{APENDICE 1}

Lista de localidades, distrito y provincias entre paréntesis, coordenadas geográficas y tipo de hábitat.

List of localities, district and province in brackets, geographic coordinates, and type of habitat.

1 km E km 25,3 de la carretera Iquitos-Nauta (Fundo San Martín) (San Juan, Loreto, Maynas) 3º 57,954’ S 73²4,259’ W-Bosque secundario

13 de Febrero, Fundo Nemith, E km 33 de la carretera Iquitos-Nauta (San Juan, Loreto, Maynas) $4^{\circ} 01,524^{\prime}$ S $73^{\circ} 25,776^{\prime}$ W-Bosque secundario

Camal Municipal de Punchana (Punchana, Loreto, Maynas) $3^{\circ} 43.568^{\prime} \mathrm{S} 73^{\circ} 14,951^{\prime} \mathrm{W}$-Área urbana

Camino a El Paujil, 1,8 km al W del km 35 de la carretera Iquitos-Nauta (San Juan, Loreto, Maynas) $4^{\circ} 01,217^{\prime} \mathrm{S} 73^{\circ} 26,787^{\prime} \mathrm{W}-\mathrm{Bosque}^{\circ}$ primario

Caserío Palo Seco, app. 500 m E km 28,8 de la carretera Iquitos-Nauta (San Juan, Loreto, Maynas) 3 59,227’ S 73² 24,920’ W-Bosque secundario

Caserío Palo Seco, 2,9 km E del km 28,8 de la carretera Iquitos-Nauta (San Juan, Loreto, Maynas) 3º 59,749’ S 73²4,359’ W-Área cultivada

CEI (Centro Educativo Inicial) Nro 165, Republica Federal de Alemania, Club de Leones (Punchana, Loreto, Maynas) $3^{\circ} 43,728^{\prime} \mathrm{S} 73^{\circ}$ 14,704 ' W-Área urbana

Comunidad de Manacamiri, Río Nanay, Fundo Morropon (Punchana, Loreto, Maynas) 3 42,594’ S 73º 18,170’ W—Bosque primario

Corrientillo, km 6 de la carretera Iquitos-Nauta, 4,5 km W camino a Zungarococha (San Juan, Loreto, Maynas) $3^{\circ} 49,955^{\prime} \mathrm{S} 73^{\circ} 21,750^{\prime}$ $\mathrm{W}$-Zona rural

El Dorado, km 25 de la carretera Iquitos-Nauta, app. 1,5 km al E (San Juan, Loreto, Maynas) 3 58,010’ S 73²3,620’ W-Bosque primario

El Paujil, 1,8 km al W del km 35 de la carretera Iquitos-Nauta (San Juan, Loreto, Maynas) $4^{\circ} 01,217^{\prime} \mathrm{S} 73^{\circ} 26,787^{\prime} \mathrm{W}$-Bosque primario Estación Experimental Agraria San Roque (San Juan, Loreto, Maynas) 3 47,217’ S 73º 17,695’ W-Área urbana

Ex Petroleros, 300 m W km 39,8 de la carretera Iquitos-Nauta (San Juan, Loreto, Maynas) 4 04,740’ S 73²7,180’ W—Bosque primario

Hogar "Padre Angel Rodríguez" (INABIF), Av. 28 de Julio Nro 500 (Punchana, Loreto, Maynas) 3 43,662' S 73 14,743’ W-Área urbana

Iberia, río Tapiche (Tapiche, Loreto, Requena) 5 $40^{\prime} \mathrm{S} 74^{\circ} 9^{\prime} \mathrm{W}-$ Bosque primario

Iglesia Inmaculada (Punchana, Loreto, Maynas) $3^{\circ} 43,738^{\prime}$ S $73^{\circ} 14,580^{\prime}$ W-Área urbana

Iquitos (Bermudez 1004) (Iquitos, Loreto, Maynas) 3 45,126’ S 73º 15,290’ W-Área urbana

Iquitos Oeste, CESM (Centro Educativo San Martín) “Loreto” (Iquitos, Loreto, Maynas) 3 44,697' S 73 15,770’ W—Área urbana

Iquitos, Calle Roman nro. 988 (Iquitos, Loreto, Maynas) 3ํ 45,171' S 73 15,336' W-Área urbana

Iquitos Colegio Primario Secundario“RADM” (Rosa Agustina Donayre de Morey), calle Putumayo (Iquitos, Loreto, Maynas) $3^{\circ} 44,997$ ' $\mathrm{S} 73^{\circ} 15,137^{\prime}-\mathrm{W}$ Área urbana

Iquitos, Coliseo Cerrado de Iquitos (Iquitos, Loreto, Maynas) 3 44,643’ S 73 15,235' W-Área urbana

Iquitos, Edificio EsSalud (calles Raymondi-Napo) (Iquitos, Loreto, Maynas) 3 44,942' S 73ํ 14,613' W-Área urbana

Iquitos, Villa de técnicos de la FAP (Fuerza Aérea de Perú), calle Moore última cuadra (Iquitos, Loreto, Maynas) $3^{\circ} 45,751^{\prime} \mathrm{S} 73^{\circ} 15,395^{\prime}$ $\mathrm{W}$-Área urbana

km 19 de la carretera Iquitos-Nauta (San Juan, Loreto, Maynas) $3^{\circ}$ 55,429’ S 73²2,505' W-Zona rural

Mazanillo (Belén, Loreto, Maynas) $3^{\circ} 49,685^{\prime}$ S 73 13,171’ W-Bosque primario 
Ninarumi, 7,4 km al W y 1 km al SE del km 6 de la carretera Iquitos-Nauta (San Juan, Loreto, Maynas) 3º 50,990’ S 73 $22,436^{\prime}$ WBosque secundario

Ninarumi, 7,4 km al W y 1 km al SE del km 6 de la carretera Iquitos-Nauta (San Juan, Loreto, Maynas) 3 50,990’ S 73²2,436’ W-Área cultivada

Ninarumi, 7,4 km al W y $500 \mathrm{~m}$ al SE del km 6 de la carretera Iquitos-Nauta (San Juan, Loreto, Maynas) $3^{\circ} 50,731^{\prime} \mathrm{S} 73^{\circ} 22,813^{\prime}$ WBosque secundario

Ninarumi, 8,4 km al W del km 6 de la carretera Iquitos-Nauta (San Juan, Loreto, Maynas) 3 50,576’ S 73²3,156’ W-Zona rural

Ninarumi, Colegio Nro 60778, 7,9 km al W del km 6 de la carretera Iquitos-Nauta (San Juan, Loreto, Maynas) 3 50,585’ S 73²3,102’W Zona rural

Padrecocha, 30 m de la carretera Shihua (Punchana, Loreto, Maynas) $3^{\circ} 41,544^{\prime}$ S 73 17,726' W—Bosque secundario

Paujil, W km 37,45 de la carretera Iquitos-Nauta (San Juan, Loreto, Maynas) 4 03,527' S 73²6,535' W-Bosque primario

Peña Negra, 600 m al W del km 10 de la carretera Iquitos-Nauta (San Juan, Loreto, Maynas) 3 51,322’ S 73²0,709’ W—Bosque secundario

Punchana, Bellavista Nanay (puerto) (Punchana, Loreto, Maynas) $3^{\circ} 42,231^{\prime}$ S 73 14,969' W-Área urbana

Punchana, Calle Amazonas 1086 (Punchana, Loreto, Maynas) 3 43,506' S 73 15,113' W-Área urbana

Recreo Don Pepe, al lado del cementerio de Punchana (Punchana, Loreto, Maynas) $3^{\circ} 43,365^{\prime} \mathrm{S}_{73^{\circ}} 15,351^{\prime}$ W-Área urbana

Río Nanay, Puerto Transelva (Punchana, Loreto, Maynas) 3 42,057' S 73 16,303' W-Bosque secundario

Rumococha, 3,3 km W de Av. Quiñones 4700 (San Juan, Loreto, Maynas) 3 46,651' S 73 18,793' W-Área urbana

San Juan, Piscina Olímpica del Instituto Peruano de Deportes, km 1,5 al W de la carretera Iquitos-Nauta (San Juan, Loreto, Maynas) $3^{\circ}$ 47,696' S $73^{\circ} 18,231^{\prime} \mathrm{W}$-Zona rural

San Lucas, W km 43 de la carretera Iquitos-Nauta (San Juan, Loreto, Maynas) 4º6,247’ S 732 27,791' W-Bosque primario

Varillal, 400 m W 200 m N del km 14 de la carretera Iquitos-Nauta (San Juan, Loreto, Maynas) 3 52,949’ S 73º 21,288’ W-Área cultivada

Recibido: 06.03.12

Aceptado: 15.05.12 